\title{
Composição corporal e exigências nutricionais de macrominerais para novilhas em crescimento das raças Guzerá e Holandesa
}

\author{
[Body composition and macrominerals nutritional requirements for growing Guzera and Holstein heifers] \\ L.S. Rabelo ${ }^{1}$, A.L.C.C. Borges ${ }^{2 *}$, L.C. Gonçalves ${ }^{2}$, G.S. Machado ${ }^{3}$, L.P. Delgado ${ }^{4}$, \\ J.J.C. Ferreira ${ }^{2}$, I. Borges ${ }^{2}$, N.M. Rodriguez ${ }^{2}$, D.S. Graça ${ }^{2}$, E.O.S. Saliba ${ }^{2}$ \\ ${ }^{1}$ Médico veterinário autônomo - Belo Horizonte, MG \\ ${ }^{2}$ Escola de Veterinária - UFMG \\ Caixa Postal 567 \\ 30123-970 - Belo Horizonte, MG \\ ${ }^{3}$ Aluno de pós-graduação - EV-UFMG - Belo Horizonte, MG \\ ${ }^{4}$ Aluno de graduação - EV-UFMG - Belo Horizonte, MG
}

\begin{abstract}
RESUMO
Foram utilizadas 22 novilhas, 11 da raça Holandesas e 11 da Guzerá, com peso vivo (PV) inicial de $189 \mathrm{~kg}$. Após o abate inicial de seis animais de cada raça, outros dois grupos, cinco animais de cada raça, passaram a receber dieta à base de feno de Tifton 85 (Cynodon spp.), milho, farelo de soja e mistura mineral, para ganho diário de $1 \mathrm{~kg}$ de peso vivo (PV). O abate final ocorreu após ganho de peso de $100 \mathrm{~kg}$. Durante os dois abates, todos os tecidos foram pesados e amostrados, retirando-se como amostra representativa da carcaça entre a nona e a décima primeira costela para determinação dos conteúdos corporais de $\mathrm{Ca}, \mathrm{P}, \mathrm{Mg}, \mathrm{Na}$ e $\mathrm{K}$. Determinaram-se a composição corporal e as exigências nutricionais desses minerais para ganho de $1 \mathrm{~kg}$ de PV. Houve decréscimo na concentração dos cinco macrominerais no peso de corpo vazio e no peso ganho com a elevação do PV. Não houve diferença entre as raças quanto às exigências nutricionais dos macrominerais. As exigências dietéticas totais de $\mathrm{Ca}, \mathrm{P}, \mathrm{Mg}, \mathrm{Na}$ e $\mathrm{K}$ para animais de $200 \mathrm{~kg}$ de PV foram de 11,$57 ; 5,97 ; 0,36 ; 1,30$ e $0,86 \mathrm{~g} /$ dia, respectivamente.
\end{abstract}

Palavras-chave: bovino, cálcio, fósforo, magnésio, potássio, sódio

\begin{abstract}
Twenty-two heifers, 11 Holstein and 11 Guzera, with initial live weight (LW) of $189 \mathrm{~kg}$ were used in this study. Six animals of each breed were slaughtered (treatment $R$ ), and two groups of five animals of each breed were fed ad libitum diet of Tifton 85 (Cynodon spp.) hay, ground corn, soybean meal and mixture minerals, for a daily gain of $1 \mathrm{~kg}$. The animals were slaughtered after reaching weight gain of $100 \mathrm{~kg}$. All tissues were weighted and representative samples were taken from the ninth to eleventh rib, and analyzed for body contents of $\mathrm{Ca}, \mathrm{P}, \mathrm{Mg}, \mathrm{Na}$ and $\mathrm{K}$. The body composition and the mineral requirements for a $1 \mathrm{~kg}$ $L W$ daily weight gain were determined. Decreases in the concentration of macrominerals in the empty body weight and in carcass weight gain were related to $L W$ increased. No differences among breeds for macrominerals were observed. The $\mathrm{Ca}, \mathrm{P}, \mathrm{Mg}, \mathrm{Na}$ and $\mathrm{K}$ dietary requirements for animals of $200 \mathrm{~kg}$ of $L W$ were $11.57 ; 5.97 ; 0.36 ; 1.30$ and $0.86 g /$ day, respectively.
\end{abstract}

Keywords: cattle, calcium, magnesium, phosphorus, potassium, sodium

Recebido em 30 de julho de 2005

Aceito em 18 de dezembro de 2006

*Autor para correspondência (corresponding author)

E-mail: analuiza@vet.ufmg.br

Apoio: FAPEMIG 


\section{INTRODUÇÃO}

Os elementos minerais estão presentes nas células e tecidos animais e possuem uma grande variedade de combinações químicas e funcionais. Suas concentrações são características e apresentam variações de acordo com o elemento e com o tecido (Morais et al., 2000).

Fatores como espécie, idade, sexo, raça, peso e nível de ingestão de energia influenciam essas variações e conduzem a diferenças nas exigências nutricionais dos animais (Garrett, 1980), tornando-se necessário avaliar a composição corporal para determinação das suas exigências nutricionais (Borges, 2000).

Considerando-se animais pertencentes à mesma raça e com peso de corpo vazio equivalente, fêmeas têm maior quantidade corporal de gordura que machos castrados, e esses mais do que os machos inteiros (Lana et al., 1992; Borges, 2000). Desse modo, a retenção de minerais depende da composição do ganho de peso, sendo que maiores deposições de gordura reduzem as deposições de elementos inorgânicos, uma vez que as concentrações de minerais no tecido adiposo são menores que nos tecidos muscular e ósseo (Fontes, 1995).

O Reappraisal... (1991) e o Nutrient... (2001) consideraram que o crescimento ósseo diminui com a maturidade e adotaram um outro modelo onde as exigências líquidas de Ca e P para ganho passaram a ser estimadas em função do peso à maturidade e do peso vivo do animal. O Nutrient... (1984, 1996) calcula as exigências líquidas de Ca e P para ganho em função da taxa de deposição de proteína.

No Brasil, a partir da década de 80 , foram desenvolvidos alguns trabalhos de pesquisa para a determinação das exigências líquidas de macrominerais em bovinos de corte, machos, de várias raças e cruzamentos. Esses trabalhos adotaram o método fatorial que consiste, por sua vez, na soma das quantidades líquidas do mineral depositadas no corpo do animal para atender ao crescimento, à engorda, à gestação, à produção de leite, ao crescimento de lã, conforme a espécie e a categoria animal, além das perdas inevitáveis do corpo, ou seja, as secreções endógenas, que são conhecidas como exigências líquidas para mantença. Secundariamente, a exigência dietética do mineral é calculada pela soma das frações para mantença e produção (exigência líquida total) dividida pelo coeficiente de absorção do elemento inorgânico no aparelho digestivo do animal.

Silva et al. (2002b) agruparam os dados sobre exigências líquidas de proteína, energia e macrominerais dos trabalhos brasileiros disponíveis até então para uma análise conjunta. Os autores observaram, em suas análises, que houve diferença entre os quatro grupos genéticos estudados (zebuínos; F1 (Europeu $\times$ Zebu); mestiços leiteiros; e Holandês) quanto aos conteúdos corporais dos elementos minerais $(\mathrm{Ca}$, $\mathrm{P}, \mathrm{Mg}, \mathrm{Na}$ e K), em função do logaritmo do peso de corpo vazio. Os resultados demonstraram, ainda, diminuição nas concentrações dos macrominerais estudados, principalmente nas de $\mathrm{Ca}, \mathrm{P}$ e Na, com o aumento do peso vivo.

Segundo Silva et al. (2002b), os animais zebuínos apresentaram as menores exigências líquidas de $\mathrm{P}, \mathrm{Mg}$ e $\mathrm{Na}$, porém maiores de $\mathrm{K}$. Os animais F1 apresentaram as maiores exigências líquidas de $\mathrm{Na}$ e $\mathrm{Ca}$, este último sendo elevado também para os animais Holandeses. As maiores exigências líquidas de $\mathrm{Mg}$ foram observadas nos mestiços leiteiros.

Quanto às fêmeas de raças de exploração leiteira, ainda não há, no Brasil, relatos de trabalhos na literatura consultada que tivessem por finalidade estudar as exigências nutricionais de macrominerais para esta categoria.

Os objetivos do presente trabalho foram determinar a composição corporal e as exigências líquidas e dietéticas dos macrominerais cálcio, fósforo, magnésio, sódio e potássio de novilhas das raças Guzerá e Holandesa em fase de crescimento, entre 200 e $300 \mathrm{~kg}$ de peso vivo.

\section{MATERIAL E MÉTODOS}

Após um período de adaptação à dieta e às condições experimentais de 70 dias, sob o mesmo tratamento, os animais foram divididos em quatro lotes, dois de cada raça, uniformes quanto ao peso vivo (PV), os quais foram designados aleatoriamente aos tratamentos: abate inicial ou referência (R) e alimentação ad libitum 
(G). No tratamento $\mathrm{R}$ foram utilizados seis animais da raça Guzerá e seis da raça Holandesa, abatidos após o período de adaptação, que serviram de referência para o estudo da composição corporal inicial. As 10 novilhas restantes foram subdivididas em dois grupos $(\mathrm{G})$, de cinco animais de cada raça, que foram alimentados com dieta ad libitum, balanceada segundo as normas do Nutrient... (1988) para ganho de PV diário de $1 \mathrm{~kg}$.

A dieta era composta de feno de Tifton 85 (Cynodon spp) e um concentrado à base de milho e farelo de soja. O feno foi picado em picadeira estacionária em partículas de aproximadamente $10 \mathrm{~cm}$. As proporções de volumoso e concentrado na ração foram de aproximadamente 55\% e $45 \%$, respectivamente, com base na matéria seca (MS). A composição percentual da ração é apresentada na Tab. 1, e os teores de MS, proteína bruta (PB), energia metabolizável (EM), carboidratos não estruturais (CNE), fibra em detergente neutro (FDN) e macrominerais encontram-se na Tab. 2.

Tabela 1. Composição percentual da ração experimental, expressa em porcentagem da matéria seca, fornecida a novilhas em crescimento

\begin{tabular}{lc}
\hline Ingredientes & Porcentagem \\
\hline Feno & 55,00 \\
Milho (grão moído) & 30,25 \\
Farelo de soja & 13,00 \\
Calcário calcítico $^{\text {Fosfato bicálcico }}$ & 0,81 \\
Suplemento mineral $^{1}$ & 0,13 \\
Cloreto de sódio $(\mathrm{NaCl})$ & 0,36 \\
\hline
\end{tabular}

${ }^{1}$ Suplemento mineral contendo em um quilograma: $\mathrm{Ca}=180 \mathrm{~g}, \mathrm{P}=130 \mathrm{~g}, \mathrm{Cu}=1.250 \mathrm{mg}, \mathrm{Zn}=3.600 \mathrm{mg}$, $\mathrm{Mn}=2.000 \mathrm{mg}, \mathrm{Co}=200 \mathrm{mg}, \mathrm{I}=300 \mathrm{mg}, \mathrm{Se}=10 \mathrm{mg}$, Fé $=2.200 \mathrm{mg}$.

Tabela 2. Teores médios de matéria seca (MS), proteína bruta (PB), carboidratos não estruturais $(\mathrm{CNE})$, fibra em detergente neutro $(\mathrm{FDN})$, cálcio $(\mathrm{Ca})$, fósforo $(\mathrm{P})$, magnésio $(\mathrm{Mg})$, sódio $(\mathrm{Na})$ e potássio $(\mathrm{K})$ do feno, do concentrado e da ração experimental, expressos em porcentagem da MS, e de energia metabolizável, expressa em Mcal por kg de MS, fornecidas a novilhas em crescimento

\begin{tabular}{lcccccccccc}
\hline Alimento & MS & PB & EM $^{1}$ & CNE & FDN & Ca & P & Mg & Na & K \\
\hline Feno & 87,84 & 11,99 & - & 9,53 & 65,37 & 0,43 & 0,26 & 0,12 & 0,09 & 1,30 \\
Concentrado & 88,92 & 20,00 & - & 64,64 & 16,77 & 0,81 & 0,57 & 0,26 & 0,29 & 1,08 \\
Ração experimental & 88,33 & 15,59 & 2,09 & 34,33 & 43,50 & 0,60 & 0,40 & 0,18 & 0,18 & 1,20 \\
\hline
\end{tabular}

A ração foi fornecida duas vezes ao dia, às oito e às 15 horas, em quantidades diariamente ajustadas de forma a manter as sobras do grupo $\mathrm{G}$ em torno de $10 \%$ do ofertado. As sobras foram pesadas e amostradas diariamente, às sete horas, e congeladas em freezer a $-10^{\circ} \mathrm{C}$. As amostras de concentrado e feno foram coletadas semanalmente para análises químicas posteriores.

O período experimental não teve duração preestabelecida. Os animais foram abatidos quando os dois grupos $(\mathrm{G})$ atingiram ganho de PV médio de $100 \mathrm{~kg}$, o que correspondeu ao PV final próximo de $300 \mathrm{~kg}$.

A pesagem inicial foi feita por três dias consecutivos para determinação do PV inicial médio. As pesagens intermediárias foram feitas a cada 14 dias, sempre no mesmo horário. Antes de cada abate os animais foram submetidos a um jejum de 20 horas. Os animais foram sacrificados conforme rotina de frigorífico utilizando-se uma pistola de ar comprimido, seguindo-se a sangria total. A amostragem dos tecidos foi realizada como descrita por Borges (2000). O sangue foi amostrado e seu peso total foi estimado segundo Hansard et al. (1952). As duas meias carcaças foram pesadas logo após o abate. Uma amostra representativa da carcaça esquerda foi retirada, correspondente à seção da $9^{\mathrm{a}}$ à $11^{\mathrm{a}}$ costelas (seção HH), segundo Hankins e Howe (1946), para posterior dissecação, avaliação dos componentes físicos e análises de laboratório.

As soluções minerais das amostras prédesengorduradas de músculo, gordura e osso da seção HH, cabeça, canela, cauda, couro, composta de vísceras e composta de órgãos foram preparadas por via seca, segundo Silva e Queiroz (2002). De acordo com as metodologias propostas por esses autores, os teores de $\mathrm{P}$ foram determinados por colorimetria em espectrofotômetro ${ }^{1}$, ajustado para 650 nanômetros de comprimento de onda; os teores

${ }^{1} \mathrm{CELM}^{\circledast}(\mathrm{E}-225 \mathrm{D})$ - Barueri, SP - Brasil 
de $\mathrm{Ca}$ e $\mathrm{Mg}$ foram determinados em espectrofotômetro ${ }^{2}$ de absorção atômica, e os de $\mathrm{Na}$ e K, em fotômetro ${ }^{3}$ de chama.

Os teores de minerais no sangue foram estimados com base nos dados de referência para os componentes do sangue total de bovinos, cujos valores são: 7,0;18,0;4,0;260,0 e 40,0mg/100ml de sangue, para o $\mathrm{Ca}, \mathrm{P}, \mathrm{Mg}, \mathrm{Na}$ e K, respectivamente. Considerou-se o valor de 1,052 como sendo o peso específico do sangue de bovinos.

Com base nas proporções de músculo, gordura e ossos na seção $\mathrm{HH}$, determinou-se a proporção deles na carcaça, segundo as equações desenvolvidas por Hankins e Howe (1946).

O PCVZ foi determinado pela soma do peso da carcaça, rúmen-retículo, omaso, abomaso, intestino delgado, intestino grosso, mesentério, gordura interna, sangue, fígado, coração, rins, baço, pulmão, língua, couro, esôfago, traquéia, aparelho gênito-urinário, pés, cabeça e cauda. A relação entre o PCVZ e o PV dos animais do grupo $\mathrm{R}$ de cada raça foi utilizada para estimar o PCVZ inicial dos animais da mesma raça, que foram abatidos posteriormente.

Os conteúdos de $\mathrm{Ca}, \mathrm{P}, \mathrm{Mg}, \mathrm{Na}$ e $\mathrm{K}$ retidos no corpo dos animais de cada grupamento racial e tratamento foram estimados por meio de equações de regressão do logaritmo do conteúdo corporal de $\mathrm{Ca}, \mathrm{P}, \mathrm{Mg}$, $\mathrm{Na}$ ou $\mathrm{K}$, em função do logaritmo do PCVZ, segundo o Nutrient... (1980), conforme o seguinte modelo:

$\mathrm{Y}=\mathrm{a}+\mathrm{bX}+\mathrm{e}$, em que:

$\mathrm{Y}=$ logaritmo do conteúdo total, em $\mathrm{kg}$, de $\mathrm{Ca}, \mathrm{P}$, $\mathrm{Mg}$, $\mathrm{Na}$ ou $\mathrm{K}$ retido no corpo vazio;

$\mathrm{a}=$ constante;

$\mathrm{b}=$ coeficiente de regressão do logaritmo do conteúdo de $\mathrm{Ca}, \mathrm{P}, \mathrm{Mg}, \mathrm{Na}$ ou $\mathrm{K}$, em relação ao logaritmo do PCVZ;

$\mathrm{X}=$ logaritmo do PCVZ; e

$\mathrm{e}=$ erro aleatório.

Derivando-se as equações de predição do conteúdo de $\mathrm{Ca}, \mathrm{P}, \mathrm{Mg}, \mathrm{Na}$ ou $\mathrm{K}$, em função do logaritmo do PCVZ, foram obtidas as equações de predição das exigências líquidas de $\mathrm{Ca}, \mathrm{P}$,

${ }^{2}$ VARIAN $^{\circledR}$ (SpectrAA-220) - Palo Alto, Calif - EUA

${ }^{3} \mathrm{CELM}^{\circledR}$ (FC-280) - Barueri, SP - Brasil
$\mathrm{Mg}$, Na e K para ganho de $1 \mathrm{~kg}$ de $\mathrm{PCVZ}$, do tipo:

$\mathrm{Y}^{\prime}=\mathrm{b} \times 10^{\mathrm{a}} \times \mathrm{X}^{\mathrm{b}-1}$, em que:

$\mathrm{Y}^{\prime}=$ exigência líquida de $\mathrm{Ca}, \mathrm{P}, \mathrm{Mg}, \mathrm{Na}$ ou $\mathrm{K}$ (em $\mathrm{kg})$;

a e $b=$ intercepto e coeficiente de regressão, respectivamente, das equações de predição dos conteúdos corporais de $\mathrm{Ca}, \mathrm{P}, \mathrm{Mg}, \mathrm{Na}$ ou $\mathrm{K}$; e $\mathrm{X}=\mathrm{PCVZ}(\mathrm{em} \mathrm{kg})$.

Para a conversão do PV em PCVZ, dentro do intervalo de pesos em estudo, utilizou-se a relação obtida entre o PCVZ de todos os animais estudados (equação geral), em função dos respectivos $\mathrm{PV}$, segundo a equação $\mathrm{PCVZ}=8,8618185+$ $0,82705 \mathrm{PV}\left(\mathrm{R}^{2}=0,96\right)$, e para conversão das exigências líquidas para ganho de PCVZ em exigências líquidas para ganho de $\mathrm{PV}$, adotou-se a divisão pelo fator 1,2091, obtido a partir dos dados experimentais analisados para todos os animais em conjunto, conforme Borges (2000).

Para estimar as exigências líquidas de mantença foram adotadas as recomendações do Nutrient... (1980) e do Reappraisal... (1991) para perdas endógenas totais de $\mathrm{Ca}, \mathrm{P}, \mathrm{Mg}, \mathrm{Na}$ e $\mathrm{K}$, e quanto ao coeficiente de absorção desses elementos nos alimentos, adotaram-se as recomendações do Nutrient... (1980) e do Nutrient... (1996), cujos parâmetros são vistos na Tab. 3 .

Para estimar a exigência dietética total dos macrominerais estudados, somou-se a exigência líquida de mantença à exigência líquida para ganho, sendo o resultado obtido dividido pelo seu respectivo coeficiente de absorção.

A determinação das equações de regressão foi feita com o auxílio do programa Microsoft Excel, constituinte do Microsoft Office 97. Utilizou-se uma equação para cada raça, usando-se os dados dos animais dos tratamentos R e G, e uma geral, abrangendo ambas as raças nos dois tratamentos. As equações obtidas foram avaliadas quanto ao coeficiente de regressão, enquanto as comparações entre grupos genéticos foram realizadas de acordo com a metodologia adotada por Regazzi (1996) para testar a identidade de modelos. Foi utilizado o SAEG 8.0 (Sistema..., 1998). Na análise de variância utilizou-se o teste de $\mathrm{F}$ a 5 ou $1 \%$ de probabilidade (Sampaio, 2002). 
Composição corporal e exigências nutricionais...

Tabela 3. Perdas endógenas totais e coeficiente de absorção de cálcio $(\mathrm{Ca})$, fósforo $(\mathrm{P})$, magnésio $(\mathrm{Mg})$, sódio $(\mathrm{Na})$ e potássio $(\mathrm{K})$ nos alimentos

\begin{tabular}{lcc}
\hline Elemento & Perda endógena total & Coeficiente de absorção $(\%)$ \\
\hline $\mathrm{Ca}(\mathrm{g} /$ dia $)$ & $\left(-0,74+0,0079 \mathrm{PV}+0,66 \mathrm{CMS}^{1}\right)^{2}$ & $50^{4}$ \\
$\mathrm{P}(\mathrm{g} /$ dia $)$ & $1,6\left(-0,06+0,693 \mathrm{CMS}^{1}\right)^{2}$ & $68^{4}$ \\
$\mathrm{Mg}$ & $3,0 \mathrm{mg} /{\mathrm{kgPV} / \mathrm{dia}^{3}}^{3}$ & $17^{3}$ \\
$\mathrm{Na}$ & $6,8 \mathrm{mg} / \mathrm{kgPV} / \mathrm{dia}^{3}$ & $91^{3}$ \\
$\mathrm{~K}$ & & $100^{3}$ \\
$\mathrm{~K}-\quad$ Fecal & $2,6 \mathrm{~g} / \mathrm{kg} \mathrm{CMS}^{3}$ & \\
$\mathrm{~K}-\quad$ Urinário & $37,5 \mathrm{mg} / \mathrm{kgPV}^{3}$ & \\
$\mathrm{~K}-\quad$ Salivar & $0,7 \mathrm{~g} / 100 \mathrm{kgPV}^{3}$ & \\
$\mathrm{~K}-\quad$ Através da pele & $1,1 \mathrm{~g} / \mathrm{dia}^{3}$ & \\
\hline
\end{tabular}

${ }^{\mathrm{T}}$ Considerou-se consumo de matéria seca (CMS) de 2,01\% do PV (Borges, 2000);

${ }^{2}$ Fonte: Reappraisal... (1991);

${ }^{3}$ Fonte: Nutrient... (1980);

${ }^{4}$ Fonte: Nutrient... (1996).

\section{RESULTADOS E DISCUSSÃO}

Os parâmetros das equações de regressão do logaritmo do conteúdo corporal de $\mathrm{Ca}, \mathrm{P}, \mathrm{Mg}, \mathrm{Na}$ e K, expresso em $\mathrm{kg}$, em função do logaritmo do PCVZ, expresso em $\mathrm{kg}$, obtidas para as novilhas das raças Guzerá e Holandesa, e para todas as novilhas em conjunto (geral), estão na Tab. 4. O teste de identidade de modelos (Regazzi, 1996) indicou não haver diferença $(\mathrm{P}>0,01)$ entre as raças para nenhum dos macrominerais estudados, tendo sido adotada a equação geral para a apresentação dos resultados.

Tabela 4. Parâmetros das equações de regressão do logaritmo do conteúdo corporal de cálcio, fósforo, magnésio, sódio e potássio $(\mathrm{kg})$ em função do logaritmo do peso do corpo vazio $(\mathrm{kg})$, para novilhas das raças Guzerá e Holandesa, e seus respectivos coeficientes de determinação $\left(\mathrm{R}^{2}\right)$ e coeficientes de variação $(C V)$

\begin{tabular}{|c|c|c|c|c|}
\hline \multirow{2}{*}{ Raça } & \multicolumn{2}{|c|}{ Parâmetros } & \multirow{2}{*}{$\mathrm{R}^{2}$} & \multirow{2}{*}{$\mathrm{CV}(\%)$} \\
\hline & Intercepto (a) & Coeficiente (b) & & \\
\hline \multicolumn{5}{|c|}{ Cálcio } \\
\hline Guzerá & $-0,7588373$ & $0,5997817^{*}$ & 0,79 & 6,44 \\
\hline Holandesa & $-0,3714771$ & $0,4331325^{*}$ & 0,58 & 7,44 \\
\hline Geral & $-0,5614023$ & $0,5149993^{*}$ & 0,68 & 6,91 \\
\hline \multicolumn{5}{|c|}{ Fósforo } \\
\hline Guzerá & $-0,9994416$ & $0,5791313^{*}$ & 0,80 & 11,80 \\
\hline Holandesa & $-0,6963915$ & $0,4514245^{*}$ & 0,60 & 14,19 \\
\hline Geral & $-0,8417791$ & $0,5127076^{*}$ & 0,69 & 12,97 \\
\hline \multicolumn{5}{|c|}{ Magnésio } \\
\hline Guzerá & $-2,6109552$ & $0,7248505^{*}$ & 0,90 & 3,05 \\
\hline Holandesa & $-1,9772025$ & $0,4547224^{*}$ & 0,61 & 4,61 \\
\hline Geral & $-2,2849121$ & $0,5860505^{*}$ & 0,73 & 4,31 \\
\hline \multicolumn{5}{|c|}{ Sódio } \\
\hline Guzerá & $-2,4338851$ & $0,8285925^{*}$ & 0,90 & 5,92 \\
\hline Holandesa & $-2,5531801$ & $0,8829704^{*}$ & 0,97 & 3,34 \\
\hline Geral & $-2,4910286$ & $0,8545948^{*}$ & 0,94 & 4,58 \\
\hline \multicolumn{5}{|c|}{ Potássio } \\
\hline Guzerá & $-2,2454418$ & $0,6947474^{*}$ & 0,75 & 7,38 \\
\hline Holandesa & $-2,3181487$ & $0,7391012^{*}$ & 0,78 & 7,27 \\
\hline Geral & $-2,2668358$ & $0,7101984^{*}$ & 0,74 & 7,27 \\
\hline
\end{tabular}

"Significativo $(\mathrm{P}<0,01)$, teste de $\mathrm{F}$. 
Houve efeito significativo $(\mathrm{P}<0,01)$ da regressão para $\mathrm{Ca}, \mathrm{P}, \mathrm{Mg}$ e $\mathrm{K}$. Os coeficientes de determinação da equação geral mostraram, entretanto, razoável ajuste das equações aos dados. Estes valores estão de acordo com Fontes (1995) que atribuiu tal resultado às quantidades relativamente pequenas de minerais na carcaça, em comparação ao PCVZ do animal. Ferreira et al. (1998) e Veloso et al. (2002) encontraram valores de $\mathrm{R}^{2}$ para as equações de $\mathrm{Ca}, \mathrm{P}, \mathrm{Mg}$ e $\mathrm{K}$ maiores que os deste trabalho.

Quanto ao efeito do grupo racial, Lana et al. (1992), ao trabalharem com bovinos machos castrados de cinco grupos raciais (Nelore e seus mestiços F1 com Chianina e Holandês e mestiços F1 e $3 / 4$ Holandês-Gir), também não encontraram efeito do grupo genético sobre o conteúdo corporal e exigências nutricionais de $\mathrm{Ca}, \mathrm{P}, \mathrm{Mg}$, $\mathrm{Na}$ e K, não tendo, portanto, necessidade de uma equação para cada grupo genético. Silva et al. (2002b) calibraram uma equação para cada grupo genético, ao encontrarem diferenças entre os quatro grupos genéticos considerados (zebuínos, F1 Europeu corte $\times$ Zebu, mestiços leiteiros $-1 / 2$, $3 / 4$ ou 5/8 Holandês-Zebu e Holandês - fração genômica $\geq 7 / 8$ Holandês-Zebu), todos machos inteiros.

Os conteúdos corporais totais de $\mathrm{Ca}, \mathrm{P}, \mathrm{Mg}, \mathrm{Na}$ e $\mathrm{K}$, bem como suas concentrações no corpo vazio, expressas em $\mathrm{g}$ por $\mathrm{kg}$ de PCVZ, para todas as novilhas em conjunto, são apresentados na Tab. 5. Eles aumentaram com o aumento do PCVZ, nas duas raças, conforme a equação geral. A concentração dos macrominerais reduziu com o aumento do PCVZ. Esse resultado está de acordo com os obtidos por Fontes (1995), Silva et al. (2002b) e Veloso et al. (2002).

Tabela 5. Conteúdos corporais totais de cálcio $(\mathrm{Ca})$, fósforo $(\mathrm{P})$, magnésio $(\mathrm{Mg})$, sódio $(\mathrm{Na})$ e potássio (K), expressos em kg, e suas concentrações, expressas em g por kg de peso do corpo vazio (PCVZ), em novilhas das raças Guzerá e Holandesa, em conjunto, estimados pela equação geral

\begin{tabular}{|c|c|c|c|c|c|c|c|}
\hline \multirow{2}{*}{ Equação } & \multirow{2}{*}{$\begin{array}{l}\text { PV } \\
\text { (kg) }\end{array}$} & \multirow{2}{*}{$\begin{array}{c}\mathrm{PCVZ}^{1} \\
(\mathrm{~kg})\end{array}$} & \multicolumn{5}{|c|}{ Elemento } \\
\hline & & & $\mathrm{Ca}$ & $\mathrm{P}$ & $\mathrm{Mg}$ & $\mathrm{Na}$ & $\mathrm{K}$ \\
\hline \multirow{4}{*}{ Geral } & & & \multicolumn{5}{|c|}{ Conteúdo corporal total $(\mathrm{kg})$} \\
\hline & 200,00 & 174,27 & 3,916 & 2,029 & 0,107 & 0,266 & 0,211 \\
\hline & 250,00 & 215,62 & 4,370 & 2,263 & 0,121 & 0,319 & 0,246 \\
\hline & 300,00 & 256,98 & 4,783 & 2,476 & 0,134 & 0,370 & 0,278 \\
\hline \multicolumn{8}{|c|}{ Concentração corporal (g/kg PCVZ) } \\
\hline \multirow{4}{*}{ Geral } & 200,00 & 174,27 & 22,47 & 11,64 & 0,61 & 1,53 & 1,21 \\
\hline & 231,11 & 200,00 & 21,02 & 10,88 & 0,58 & 1,49 & 1,16 \\
\hline & 250,00 & 215,62 & 20,27 & 10,50 & 0,56 & 1,48 & 1,14 \\
\hline & 300,00 & 256,98 & 18,61 & 9,63 & 0,52 & 1,44 & 1,08 \\
\hline
\end{tabular}

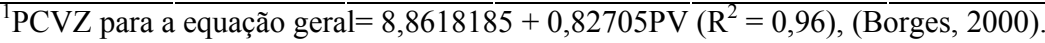

$\mathrm{PV}=$ peso vivo.

Considerando-se que as principais diferenças entre sexo envolvem a proporção de tecido adiposo, e como fêmeas têm maior quantidade corporal de gordura do que machos castrados, e estes mais do que machos inteiros de mesma raça e com PCVZ equivalente (Lana et al., 1992; Borges 2000), espera-se que as concentrações de macrominerais sejam menores em fêmeas do que em machos castrados. Os valores de concentração corporal de $\mathrm{Ca}$ e $\mathrm{Mg}$ foram, em média, 5,9\% superiores aos valores encontrados por Fontes (1995) para animais machos castrados com PCVZ equivalentes, e menores em relação aos animais inteiros para os cinco macrominerais. Essas diferenças foram da ordem de 18,$2 ; 15,5 ; 11,8 ; 3,7$ e $8,8 \%$, respectivamente, para $\mathrm{Ca}, \mathrm{P}, \mathrm{Mg}, \mathrm{Na}$ e K. O valor da concentração corporal de $\mathrm{Na}$ foi $6,7 \%$ maior do que o obtido para animais castrados de $200 \mathrm{~kg}$ de PCVZ, estudados por Fontes (1995). Assim, estes resultados contrastam com o esperado, pois de acordo com a literatura consultada, haveria menor concentração corporal de minerais em fêmeas. Uma possível explicação para essas divergências poderia ser dada pela diferença de maturidade fisiológica dos animais estudados por Fontes (1995), que se encontravam em fase de terminação, sendo, portanto, mais velhos que os animais deste experimento, que se encontravam em fase de crescimento, além de pertencerem a 
grupos genéticos distintos. Para o $\mathrm{P}$ e o $\mathrm{K}$, os valores foram 8,4 e $56,9 \%$ menores do que as concentrações relatadas por aquele autor.

Derivando-se as equações de regressão do logaritmo do conteúdo corporal de $\mathrm{Ca}, \mathrm{P}, \mathrm{Mg}, \mathrm{Na}$ e K em função do logaritmo do PCVZ, foram obtidas as equações de predição das exigências líquidas de $\mathrm{Ca}, \mathrm{P}, \mathrm{Mg}, \mathrm{Na}$ e $\mathrm{K}$ por $\mathrm{kg}$ de ganho de PVCZ (Tab. 6). Os conteúdos encontrados desses macrominerais referem-se às exigências líquidas para ganho de $1 \mathrm{~kg}$ de PCVZ.

As exigências líquidas de $\mathrm{Ca}, \mathrm{P}, \mathrm{Mg}, \mathrm{Na}$ e K para ganho de $1 \mathrm{~kg}$ de PCVZ para animais de 200, 230,250 e $300 \mathrm{~kg}$ de PV, obtidas a partir das equações derivadas para todas as novilhas em conjunto, bem como as exigências convertidas para ganho de $1 \mathrm{~kg}$ de $\mathrm{PV}$, são apresentadas na Tab. 7. Para a transformação em ganho de PV, as exigências líquidas para ganho de $1 \mathrm{~kg}$ PCVZ foram divididas pelo fator 1,2091, obtido por
Borges (2000), para todas as novilhas em conjunto utilizadas neste experimento.

Tabela 6. Equações de predição das exigências líquidas de cálcio, fósforo, magnésio, sódio e potássio por $\mathrm{kg}$ de ganho de peso do corpo vazio novilhas das raças Guzerá e Holandesa

Elemento Equação Equação de predição ${ }^{1}$

\begin{tabular}{|c|c|c|}
\hline Cálcio & Geral & $\mathrm{y}^{\prime}=\underset{0,485001}{0,141385 \times \mathrm{PCVZ}}$ \\
\hline Fósforo & Geral & $\mathrm{y}^{\prime}=\underset{0,487292}{0,073806} \times \mathrm{PCVZ}$ \\
\hline Magnésio & Geral & $\mathrm{y}^{\prime}=\underset{0,413950}{0,003041 \times \mathrm{PCVZ}}$ \\
\hline Sódio & Geral & $\mathrm{y}^{\prime}=\underset{0,145405}{\underset{0}{0} 002759} \times \mathrm{PCVZ}$ \\
\hline Potássio & Geral & $\mathrm{y}^{\prime}=\underset{0,289802}{0,003842} \times \mathrm{PCVZ}$ \\
\hline
\end{tabular}

${ }^{1} \mathrm{PCVZ}=$ peso do corpo vazio, expresso em $\mathrm{kg}$.

Tabela 7. Exigências líquidas de cálcio $(\mathrm{Ca})$, fósforo $(\mathrm{P})$, magnésio $(\mathrm{Mg})$, sódio $(\mathrm{Na})$ e potássio $(\mathrm{K})$ para ganho, expressas em g por kg de ganho de peso do corpo vazio (PCVZ) ou peso vivo (PV), obtidas por derivação das equações de novilhas das raças Guzerá e Holandesa e relação cálcio:fósforo (Ca:P)

\begin{tabular}{|c|c|c|c|c|c|c|c|c|}
\hline \multirow{2}{*}{ Equação } & \multirow{2}{*}{$\begin{array}{l}\text { PV } \\
\text { (kg) }\end{array}$} & \multirow{2}{*}{$\begin{array}{c}\text { PCVZ } \\
(\mathrm{kg})\end{array}$} & \multicolumn{5}{|c|}{ Exigência líquida para ganho } & \multirow{2}{*}{$\begin{array}{c}\text { Relação } \\
\text { Ca:P }\end{array}$} \\
\hline & & & $\mathrm{Ca}$ & $\mathrm{P}$ & $\mathrm{Mg}$ & $\mathrm{Na}$ & $\mathrm{K}$ & \\
\hline \multicolumn{9}{|c|}{$\mathrm{g} / \mathrm{kg}$ de ganho de $\mathrm{PCVZ}$} \\
\hline \multirow{4}{*}{ Geral } & 200,00 & 174,27 & 11,57 & 5,97 & 0,36 & 1,30 & 0,86 & 1,94 \\
\hline & 231,11 & 200,00 & 10,82 & 5,58 & 0,34 & 1,27 & 0,83 & 1,94 \\
\hline & 250,00 & 215,62 & 10,44 & 5,38 & 0,33 & 1,26 & 0,81 & 1,94 \\
\hline & 300,00 & 256,98 & 9,59 & 4,94 & 0,31 & 1,23 & 0,77 & 1,94 \\
\hline \multirow{5}{*}{ Geral } & & \multicolumn{7}{|c|}{$\mathrm{g} / \mathrm{kg}$ de ganho de $\mathrm{PV}$} \\
\hline & 200,00 & 174,27 & 9,57 & 4,94 & 0,30 & 1,08 & 0,71 & 1,94 \\
\hline & 231,11 & 200,00 & 8,95 & 4,62 & 0,28 & 1,05 & 0,69 & 1,94 \\
\hline & 250,00 & 215,62 & 8,63 & 4,45 & 0,27 & 1,04 & 0,67 & 1,94 \\
\hline & 300,00 & 256,98 & 7,93 & 4,09 & 0,26 & 1,02 & 0,64 & 1,94 \\
\hline
\end{tabular}

Da mesma forma como a concentração corporal, as exigências líquidas dos macrominerais por $\mathrm{kg}$ de ganho de PCVZ também apresentaram redução com o aumento do peso corporal dos animais. Estes resultados assemelham-se aos de Paulino et al. (1999), Silva et al. (2002a) e Silva et al. (2002b).

As exigências líquidas dos cinco macrominerais, tanto para ganho de $1 \mathrm{~kg}$ de PCVZ quanto para $1 \mathrm{~kg}$ de PV, estão abaixo das exigências encontradas por pesquisas brasileiras (Signoretti et al., 1999; Veloso et al., 2002), que estudaram novilhos inteiros de diversos grupos genéticos, mas com PCVZ semelhantes aos deste trabalho.

As exigências líquidas de $\mathrm{Ca}$ e $\mathrm{P}$ para ganho encontradas são inferiores aos valores sugeridos pelo Nutrient... (1980).

O Nutrient... (1984, 1996) adota valores de 7,1 e $3,9 \mathrm{~g} / 100 \mathrm{~g}$ de proteína retida como sendo as exigências líquidas de $\mathrm{Ca}$ e $\mathrm{P}$, respectivamente. Borges (2000), que trabalhou com os mesmos animais deste experimento, ao estudar as exigências nutricionais de proteína e energia, encontrou deposições de proteína de 128,4 e 122, 
$7 \mathrm{~g} / \mathrm{kg}$ de PV para animais de 200 e $300 \mathrm{~kg}$ PV, respectivamente. A partir desses dados têm-se relações de $7,7 \mathrm{~g}$ de $\mathrm{Ca}$ e $3,8 \mathrm{~g}$ de $\mathrm{P}$ por $100 \mathrm{~g}$ de proteína retida para animais de $200 \mathrm{~kg}$ de $\mathrm{PV}$. Para animais de $300 \mathrm{~kg}$ de PV, têm-se relações de $6,4 \mathrm{~g}$ Ca e $3,3 \mathrm{~g}$ de $\mathrm{P}$ por $100 \mathrm{~g}$ de proteína. Assim, as exigências líquidas de $\mathrm{Ca}$ e $\mathrm{P}$ encontradas foram próximas àquelas propostas pelo Nutrient... (1984, 1996) para animais de $200 \mathrm{~kg}$ de PV e inferiores às exigências para animais de $300 \mathrm{~kg}$ de PV.

Os resultados estão próximos aos recomendados pelo Reappraisal... (1991), que propôs equações com base no crescimento ósseo, nas quais ficou demonstrada redução das exigências líquidas de $\mathrm{Ca}$ e $\mathrm{P}$, por $\mathrm{kg}$ de ganho de $\mathrm{PV}$, à medida que o $\mathrm{PV}$ do animal se eleva. O mesmo acontece em relação ao Nutrient... (2001), que adotou o modelo proposto pelo Reappraisal... (1991). Os valores de exigências líquidas sugeridos pelo Reappraisal... (1991) e Nutrient... (2001) para um animal de $200 \mathrm{~kg}$ de $\mathrm{PV}$, com peso à maturidade de $600 \mathrm{~kg}$ e com taxa de ganho de $1 \mathrm{~kg}$ de PV por dia, são de 12,5 e 7,1g/dia de $\mathrm{Ca}$ e de $\mathrm{P}$, respectivamente. Para um animal de $300 \mathrm{~kg}$ essas exigências diminuem para 11,4 e $6,6 \mathrm{~g} / \mathrm{dia}$, respectivamente, para o $\mathrm{Ca}$ e $\mathrm{o}$ P. Ao se comparar as exigências da Tab. $7 \mathrm{com}$ as do Reappraisal... (1991) e Nutrient... (2001) é possível notar que as exigências de $\mathrm{Ca}$ para ganho de $1 \mathrm{~kg}$ de PV são menores em 30,6 e $43,7 \%$, respectivamente, para animais de 200 e $300 \mathrm{~kg}$ de PV. Essas diferenças são ainda maiores para o $\mathrm{P}$, cujos valores são de 43,72 e $61,37 \%$, para pesos de 200 e $300 \mathrm{~kg}$, respectivamente.

As relações entre exigências líquidas de $\mathrm{Ca}: \mathrm{P}$ para ganho resultaram no valor único de 1,94 para os diferentes pesos. Essa relação é pouco maior do que a relação obtida pelo modelo do Nutrient... (1980), 1,75, e pelo modelo do Reappraisal (1991), 1,74 e 1,72 para animais de 200 e $300 \mathrm{~kg}$ de PV, respectivamente. Fontes (1995) encontrou relação $\mathrm{Ca}: \mathrm{P}$ de 1,33 para animais machos castrados em terminação com $200 \mathrm{~kg}$ de PCVZ.

Com relação ao $\mathrm{Mg}$, o Nutrient... (1980) adotou o valor $0,45 \mathrm{~g} / \mathrm{kg}$ como sendo a concentração corporal e exigência líquida desse macromineral por kg de ganho de PCVZ, independentemente do PCVZ do animal. Esse mesmo valor foi adotado pelo Nutrient... (2001) como exigência líquida de $\mathrm{Mg}$ por $\mathrm{kg}$ de ganho de peso corporal (PCVZ) de novilhas em crescimento. As concentrações corporais de $\mathrm{Mg}$ foram maiores (Tab. 5) que o valor sugerido pelo Nutrient... (1980, 2001), mas as exigências líquidas para ganho de PCVZ (Tab. 7) foram menores do que a exigência proposta pelos referidos Conselhos. Além disso, tanto a concentração quanto as exigências líquidas de $\mathrm{Mg}$ encontradas neste trabalho reduziram-se com o aumento do PCVZ dos animais, refletindo a queda da proporção de ossos (onde estão depositados cerca de $70 \%$ do $\mathrm{Mg}$ corporal) e conseqüente aumento da proporção de gordura, como observado por Borges (2000).

Para o $\mathrm{Na}$, as exigências líquidas encontram-se abaixo da sugerida pelo Nutrient... (1980), que é de $1,5 \mathrm{~g} / \mathrm{kg}$ de ganho de PCVZ, independentemente do peso do animal. O Nutrient... (2001) considerou que a exigência líquida de $\mathrm{Na}$ de animais em crescimento é de $1,40 \mathrm{~g} / \mathrm{kg}$ de ganho diário, para animais com peso entre 150 e $600 \mathrm{~kg}$ de PV. Esse valor também é maior do que os valores de exigências líquidas de $\mathrm{Na}$ encontrados neste trabalho. Silva et al. (2002b) encontraram menores exigências líquidas de $\mathrm{Na}$ por $\mathrm{kg}$ de ganho de $\mathrm{PCVZ}$ para animais machos inteiros de PV semelhantes aos deste experimento.

As exigências líquidas de $\mathrm{K}$ de $\mathrm{PCVZ}$, estimadas a partir da derivação da sua concentração corporal, situam-se entre 0,86 e $0,77 \mathrm{~g}$ para animais de 200 e $300 \mathrm{~kg}$ de $\mathrm{PV}$, respectivamente (Tab. 7). O Nutrient... (1980), baseando-se na concentração corporal, sugeriu exigência líquida de $\mathrm{K}$ igual a 2,0g, para ganho de $1 \mathrm{~kg}$ de PCVZ. Silva et al. (2002b) encontraram valores de 2,01 a 2,55g de $\mathrm{K}$ por $\mathrm{kg}$ de ganho de PCVZ para machos inteiros dos grupos genéticos Zebu e Holandês. Fontes (1995) encontrou exigência líquida de $1,41 \mathrm{~g} / \mathrm{kg}$ de ganho de PCVZ para bovinos machos castrados de $200 \mathrm{~kg}$ de PCVZ, sendo este valor $41,1 \%$ superior ao valor encontrado para os animais deste trabalho com PCVZ semelhante. As menores exigências de K encontradas para as novilhas deste experimento em relação às fontes citadas podem estar relacionadas com a menor proporção de tecido muscular (local onde está concentrada a maior parte do K corporal, segundo o Nutrient..., 2001) na carcaça das novilhas em relação às dos 
animais machos castrados, e mais ainda em relação aos machos inteiros, de mesmo PCVZ.

As exigências líquidas para mantença de $\mathrm{Ca}, \mathrm{P}$, $\mathrm{Mg}$, Na e K de novilhas de 200, 250 e $300 \mathrm{~kg}$ de PV das raças Guzerá e Holandesa em conjunto foram estimadas segundo as recomendações do Reappraisal... (1991) para Ca e P e, segundo as recomendações do Nutrient... (1980), para Mg, $\mathrm{Na}$ e K, cujos resultados estão na Tab. 8. Nota-se que essas exigências aumentam com o aumento do peso dos animais e que as exigências líquidas de mantença para o K são extremamente altas quando comparadas às exigências líquidas para ganho de peso.

$\mathrm{Na}$ Tab. 9 estão as exigências dietéticas totais onde se pode notar que elas aumentam com o PV do animal, devido à participação das exigências para mantença, que são expressas em função do PV do animal.

Tabela 8. Exigências líquidas para mantença de cálcio $(\mathrm{Ca})$, fósforo $(\mathrm{P})$, magnésio $(\mathrm{Mg})$, sódio $(\mathrm{Na})$ e potássio $(\mathrm{K})$, expressas em g por dia, de novilhas das raças Guzerá e Holandesa, entre 200 e $300 \mathrm{~kg}$ de peso vivo

\begin{tabular}{lccccccc}
\hline \multirow{2}{*}{ Equação } & $\mathrm{PV}$ & $\mathrm{PCVZ}$ & \multicolumn{5}{c}{ Exigência líquida mantença (g/dia) } \\
\cline { 5 - 8 } & $(\mathrm{kg})$ & $(\mathrm{kg})$ & $\mathrm{Ca}$ & $\mathrm{P}$ & $\mathrm{Mg}$ & $\mathrm{Na}$ & $\mathrm{K}$ \\
\hline \multirow{3}{*}{ Geral } & 200 & 173,83 & 3,49 & 4,36 & 0,60 & 1,36 & 20,45 \\
& 250 & 217,29 & 4,55 & 5,48 & 0,75 & 1,70 & 25,29 \\
& 300 & 260,74 & 5,61 & 6,59 & 0,90 & 2,04 & 30,13 \\
\hline
\end{tabular}

$\overline{\mathrm{PV}}=$ peso vivo; $\mathrm{PCVZ}=$ peso do corpo vazio.

Tabela 9. Exigências dietéticas totais (mantença $+1 \mathrm{~kg}$ de ganho de peso vivo - PV) de cálcio (Ca), fósforo $(\mathrm{P})$, magnésio $(\mathrm{Mg})$, sódio $(\mathrm{Na})$ e potássio $(\mathrm{K})$, expressas em g por dia e em porcentagem da matéria seca (\% da MS), de novilhas das raças Guzerá e Holandesa entre 200 e 300kg de peso vivo

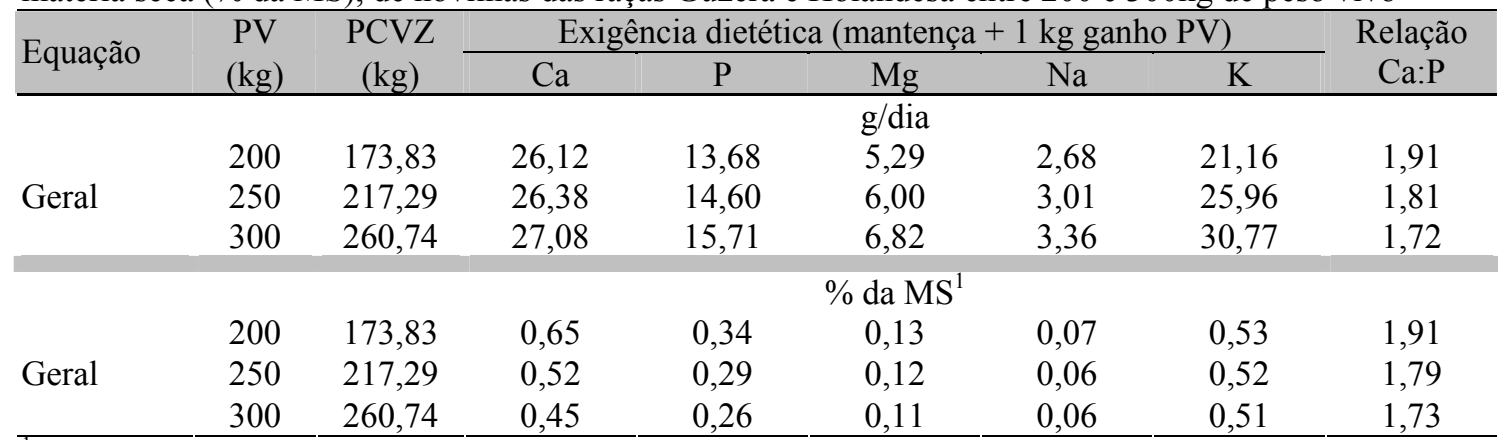

${ }^{\mathrm{T}}$ Considerando consumo médio de matéria seca igual a 2,01\% do PV (Borges, 2000).

$\mathrm{PV}=$ peso vivo; $\mathrm{PCVZ}=$ peso do corpo vazio.

A exigência dietética total de Ca para animais de $200 \mathrm{~kg}$ de PV foi superior em 6,6 a 16,8\%, em média, em relação às mesmas exigências recomendadas pelo Nutrient... (1980, 1996) e Reappraisal...(1991) que são de 21,74; 24,40 e $23,52 \mathrm{~g} /$ dia, respectivamente, e $24,5 \%$ inferior à recomendação do Nutrient... (2001) que é de $34,62 \mathrm{~g} /$ dia. Esta última fonte também cita maiores exigências dietéticas totais de $\mathrm{Ca}$ para animais de $300 \mathrm{~kg}$, na ordem de $23,9 \%$ ao estimar tal exigência em 35,60 g/dia. Em ambos os casos, tais superestimativas se devem às maiores exigências líquidas para ganho $(12,5$ e $11,4 \mathrm{~g} / \mathrm{kg}$ de ganho de PV em animais de 200 e $300 \mathrm{~kg}$ de
$\mathrm{PV}$, respectivamente) e ao menor coeficiente de absorção verdadeira $(0,45)$ considerado pelo Nutrient... (2001) em relação ao coeficiente assumido neste trabalho, que foi de 0,50. A exigência dietética total de $\mathrm{Ca}$ para animais de $300 \mathrm{~kg}$ de PV encontra-se bem próxima às recomendações do Nutrient... (1988, 1996), com valores de 26,09 e $26,64 \mathrm{~g} / \mathrm{dia}$, respectivamente, enquanto o Nutrient... (1980) e o Reappraisal... (1991) parecem subestimar tal exigência ao apresentarem exigências dietéticas totais de $\mathrm{Ca}$ de 24,09 e $25,01 \mathrm{~g} /$ dia, respectivamente. 
Para as exigências dietéticas totais de $\mathrm{P}$, os valores estimados na Tab. 9 para animais de 200 e $300 \mathrm{~kg}$ de PV encontram-se mais próximos daqueles recomendados pelo Nutrient... (1984), cujos valores são 12,48 e 15,51g/dia, respectivamente. As exigências dietéticas totais de $\mathrm{P}$ são 11,8 e $8,2 \%$ superiores àquelas estimadas pelo Nutrient... (1996), cujos valores são 12,07 e 14,42g/dia, respectivamente, para animais de 200 e $300 \mathrm{~kg}$ de PV. As exigências dietéticas totais de $\mathrm{P}$ para animais de $200 \mathrm{~kg}$ estimadas pelo Nutrient...(1980, 1986, 2001) e Reappraisal...(1991) são de 15,55; 15,74; 15,99 e $19,76 \mathrm{~g} / \mathrm{dia}$, respectivamente. Os valores encontrados no presente trabalho são inferiores aos valores desses autores da ordem de 13,7; 15,$1 ; 16,9$ e $44,4 \%$, respectivamente. Para animais de $300 \mathrm{~kg}$, as exigências dietéticas são 12,$2 ; 25,6 ; 14,3$ e $44,7 \%$ inferiores ao valores propostos pelos autores citados acima, que são, respectivamente, 17,$62 ; 19,74 ; 17,95$ e $22,94 \mathrm{~g} /$ dia. A grande diferença encontrada em relação ao Reappraisal... (1991) se deve ao menor coeficiente de absorção $(0,58)$ em relação ao adotado neste experimento.

As exigências dietéticas totais de $\mathrm{Mg}$, $\mathrm{Na}$ e $\mathrm{K}$ encontradas estão próximas daquelas recomendadas pelo Nutrient... (1980) para animais de 200 e $300 \mathrm{~kg}$, em g/dia, que são, respectivamente, de 5,42 e 7,48 para o $\mathrm{Mg}, 2,96$ e 3,61 para o $\mathrm{Na}$ e 22,10 e 31,78 para o K. Para esses três macrominerais, estas pequenas diferenças encontradas em relação às recomendações do Nutrient... (1980) refletem apenas aquelas diferenças em relação às exigências líquidas para ganho corrigidas pelo coeficiente de absorção. Vale ressaltar que, conforme já observado por Fontes (1995), que as exigências líquidas de $\mathrm{Mg}$ e $\mathrm{K}$ para ganho representam apenas pequena parte das exigências dietéticas, devido à baixa disponibilidade do $\mathrm{Mg}$ da dieta e às elevadas exigências de mantença de $\mathrm{K}$.

As exigências dietéticas determinadas neste experimento, quando expressas em \% da MS, considerando que o consumo de MS foi de $2,01 \%$ do PV, conforme determinado por Borges (2000), diminuíram seus valores em relação ao aumento do peso dos animais para o $\mathrm{Ca}, \mathrm{P}, \mathrm{Mg}$ e K. O Na apresentou relação praticamente constante. Comportamento semelhante foi relatado por Silva et al. (2002b) e Veloso et al. (2002).

As exigências dietéticas de $\mathrm{Mg}$ encontram-se acima daquelas propostas pelo Nutrient... (1984, 1996), que adotou valores de $0,10 \%$ da MS, e pelo Nutrient... (1988), que adotou o valor de $0,07 \%$ da MS em dieta de bezerros.

As exigências dietéticas de $\mathrm{Na}$ e $\mathrm{K}$ estão abaixo das exigências assumidas pelo Nutrient... (1984), que apresenta valores de 0,10 e $0,65 \%$ da $\mathrm{MS}$, respectivamente. O Nutrient... (1988) recomenda $0,10 \%$ de Na na MS da dieta de vacas secas e relata ainda que a exigência dietética de $\mathrm{K}$ em dietas de novilhos em terminação estaria entre 0,50 e $0,72 \%$ da MS da dieta, estando, essa última recomendação, de acordo com o observado neste experimento. O Nutrient... (1996) assumiu valores de 0,06 a $0,08 \%$ da MS para a exigência dietética de $\mathrm{Na}$, estando os resultados encontrados neste experimento em acordo com esses valores. O Nutrient... (1996) adotou exigência dietética de $\mathrm{K}$ igual a $0,60 \%$ da MS, estando essa exigência 13,2 e 17,6\% superior aos valores encontrados para novilhas de 200 e $300 \mathrm{~kg}$ de $\mathrm{PV}$, respectivamente.

O Nutrient... (2001) adotou o modelo fatorial para o cálculo das exigências nutricionais de $\mathrm{Mg}$, $\mathrm{Na}$ e K, cujas respectivas exigências dietéticas totais, em g/dia, são de 6,08 e 7,95 para o $\mathrm{Mg}$, 5,11 e 6,89 para o $\mathrm{Na}$ e 20,15 e 30,22 para o $\mathrm{K}$, para animais de 200 e $300 \mathrm{~kg}$ de PV, respectivamente. As exigências dietéticas totais de $\mathrm{K}$ encontradas neste trabalho estão de acordo com aquelas postas acima. As exigências de $\mathrm{Mg}$ são, em média, $15,7 \%$ menores do que os do Nutrient... (2001). Para o $\mathrm{Na}$, as recomendações do Nutrient... (2001) são maiores do que as exigências dietéticas deste mineral em 90,7 e $105,1 \%$ para animais de 200 e $300 \mathrm{~kg}$ de $\mathrm{PV}$, respectivamente.

\section{CONCLUSÕES}

As exigências nutricionais de $\mathrm{Ca}, \mathrm{P}, \mathrm{Mg}, \mathrm{Na}$ e $\mathrm{K}$ não diferem entre as raças Guzerá e Holandesa para novilhas com PV entre 200 e $300 \mathrm{~kg}$. A concentração corporal $(\mathrm{g} / \mathrm{kg}$ de $\mathrm{PCVZ})$ e as exigências líquidas $(\mathrm{g} / \mathrm{kg}$ de ganho de PCVZ ou $\mathrm{PV}$ ) de $\mathrm{Ca}, \mathrm{P}, \mathrm{Mg}$, $\mathrm{Na}$ e $\mathrm{K}$ diminuem à medida que o peso dos animais se eleva. As exigências 
líquidas, estimadas pela equação geral, para ganho de $1 \mathrm{~kg}$ de PCVZ, para novilhas das raças Guzerá e Holandesa com PV entre 200 e $300 \mathrm{~kg}$, variam de 11,57 a $9,59 \mathrm{~g}$ para o $\mathrm{Ca} ; 5,97$ a 4,94g para o $\mathrm{P} ; 0,36$ a $0,31 \mathrm{~g}$ para o $\mathrm{Mg} ; 1,30$ a 1,23 para o Na; e 0,86 a 0,77 para o K. As exigências dietéticas totais, estimadas pela equação geral, para ganho de $1 \mathrm{~kg}$ de $\mathrm{PV}$, para novilhas das raças Guzerá e Holandesa com PV entre 200 e $300 \mathrm{~kg}$, variam de 26,12 a $27,08 \mathrm{~g}$ para o $\mathrm{Ca}$; 13,68 a $15,71 \mathrm{~g}$ para o $\mathrm{P} ; 5,29$ a $6,82 \mathrm{~g}$ para $\mathrm{p} \mathrm{Mg}$; 2,68 a 3,36 g para o $\mathrm{Na}$; e 21,16 a $30,77 \mathrm{~g}$ para o $\mathrm{K}$.

\section{REFERÊNCIAS BIBLIOGRÁFICAS}

BORGES, A.L.C.C. Exigências nutricionais de proteina e energia de novilhas das raças Guzerá e Holandesa. 2000. 90f. Tese (Doutorado em Ciência Animal) - Escola de Veterinária, Universidade Federal de Minas Gerais, Belo Horizonte.

FERREIRA, M.A.; VALADARES FILHO, S.C.; COELHO DA SILVA, J.F. et al. Consumo, conversão alimentar, ganho de peso e características de carcaça de bovinos F1 Simental e Nelore. Rev. Bras. Zootec., v.28, p.352-360, 1998.

FONTES, C.A.A. Composição corporal, exigências líquidas de nutrientes para ganho de peso e desempenho produtivo de animais zebuínos e mestiços europeu-zebu: resultados experimentais. In: SIMPÓSIO INTERNACIONAL SOBRE EXIGÊNCIAS NUTRICIONAIS DE RUMINANTES, 1995, Viçosa, MG. Anais... Viçosa, MG: CARD, 1995. p.419-455.

GARRETT, W.N. Factors influencing energetic efficiency of beef production. J. Anim. Sci., v.51, p.1434-1440, 1980

HANKINS, O.G.; HOWE, P.E. Estimation of the composition of beef carcasses and cuts. s.e. Washington, D.C. USDA, 1946. (Technical Bulletin - USDA, 926).

HANSARD, S.L.; BUTLER, W.O; COMAR, C.L. et al. Blood volume of farm animals. J. Anim. Sci., v.12, p.402413, 1952.

LANA, R.P.; FONTES, C.A.A.; PERÓN, A.J. et al. Composição corporal e do ganho de peso e exigências de energia, proteína e macroelementos minerais $(\mathrm{Ca}, \mathrm{P}, \mathrm{Mg}, \mathrm{Na}$ e K) de novilhos de cinco grupos raciais. 3. Conteúdo corporal e do ganho de peso e exigências de macroelementos minerais. Rev. Bras. Zootec., v.21, p.538-549, 1992.

MORAIS, M.G.; GONÇALVES, L.C.; LOPES, H.O.S. et al. Variação sazonal de eletrólitos no sangue de vacas aneloradas sob pastejo contínuo de Brachiaria decumbens. Arq. Bras. Med. Vet. Zootec., v.52, p.105-111, 2000.
NUTRIENT requeriments of beef cattle. 6.ed. Washington, DC: National Academy Press. 1984. 90p.

NUTRIENT requeriments of beef cattle. 7.ed. Washington, DC: National Academy of Sciences, 1996. 242p.

NUTRIENT requirements of dairy cattle. 6.ed. Washington, DC: National Academy of Sciences, 1988. 158p.

NUTRIENT requeriments of dairy cattle. 7.ed. Washington, DC: National Academy of Sciences, 2001. 381p.

NUTRIENT requeriments of ruminants livestock. London: Commonwealth Agricultural Bureaux International, 1980. $351 \mathrm{p}$

OWENS, F.N.; DUBESKI, P.; HANSON, C.F. Factors that alter the growth and development of ruminants. J. Anim. Sci. v.71, p.3138-3150, 1993.

PAULINO, M.F.; FONTES, C.A.A.; JORGE, A.M. et al. Composição corporal e exigências de macroelementos minerais (Ca, P, Mg, Na e K) de bovinos não-castrados de quatro raças zebuínas. Rev. Bras. Zootec., v.28, p.634-641, 1999.

REAPPRAISAL of the calcium and phosphorus requirements of sheep and cattle. Nut. Abstr. Rev. Series B., v.61, p.576$612,1991$.

REGAZZI, J.A. Teste para verificar a identidade de modelos de regressão. Pesq. Agropec. Bras., v.31, p.1-17, 1996.

SAMPAIO, I.B.M. Estatística aplicada à experimentação animal. Belo Horizonte: Fundação de Ensino e Pesquisa em Medicina Veterinária e Zootecnia, 2002. 265p.

SIGNORETTI, R.D.; COELHO DA SILVA, J.F.; VALADARES FILHO, S.C. et al. Composição corporal e exigências líquidas e dietéticas de macroelementos inorgânicos $(\mathrm{Ca}, \mathrm{P}, \mathrm{Mg}, \mathrm{K}$ e $\mathrm{Na})$ de bezerros da raça Holandesa alimentados com dietas contendo diferentes níveis de concentrado. Rev. Bras. Zootec., v.28, p.205-213, 1999.

SILVA, D.J; QUEIROZ, A.C. Análise de alimentos (métodos químicos e biológicos). Viçosa, MG: UFV, 2002. 235p.

SILVA, F.F.; VALADARES FILHO, S.C.; ÍTALO, L.C.V. et al. Composição corporal e requisitos líquidos e dietéticos de macroelementos minerais de bovinos Nelore não-castrados. Rev. Bras. Zootec., v.31, p.757-764, 2002a.

SILVA, F.F.; VALADARES FILHO, S.C.; ÍTALO, L.C.V. et al. Exigências líquidas e dietéticas de energia, proteína e macroelementos minerais de bovinos de corte no Brasil. Rev. Bras. Zootec., v.31, p.776-792, 2002 b.

SISTEMA de análises estatísticas e genéticas. SAEG 8.0. Viçosa: UFV, 1998

VELOSO, C.M.; VALADARES FILHO, S.C.; GESUALDI JR., A. et al. Composição corporal e exigências líquidas e dietéticas de macroelementos minerais de bovinos F1 Limousin x Nelore não castrados. Rev. Bras. Zootec., v.31, p.1294-1301, 2002. 